\title{
Tetherin and Its Viral Antagonists
}

\author{
Björn D. Kuhl • Vicky Cheng • Mark A. Wainberg • \\ Chen Liang
}

Received: 2 November 2010 / Accepted: 27 December 2010 /Published online: 11 January 2011

(C) The Author(s) 2011. This article is published with open access at Springerlink.com

\begin{abstract}
Restriction factors comprise an important layer of host defense to fight against viral infection. Some restriction factors are constitutively expressed whereas the majority is induced by interferon to elicit innate immunity. In addition to a number of well-characterized interferoninducible antiviral factors such as RNaseL/OAS, ISG15, Mx, PKR, and ADAR, tetherin (BST-2/CD317/HM1.24) was recently discovered to block the release of enveloped viruses from the cell surface, which is regarded as a novel antiviral mechanism induced by interferon. Here, we briefly review the history of tetherin discovery, discuss how tetherin blocks virus production, and highlight the viral countermeasures to evade tetherin restriction.
\end{abstract}

Keywords HIV-1 · Tetherin · Vpu · Virus release · Interferon

\section{Introduction}

Interferon triggers innate immunity to protect cells from viral infection. Production of interferon is initiated with recognition of viral components, mainly viral nucleic acids,

Björn D. Kuhl and Vicky Cheng contributed equally to this work.

B. D. Kuhl $\cdot$ V. Cheng $\cdot$ M. A. Wainberg $\cdot$ C. Liang $(\bowtie)$

McGill AIDS Centre,

Lady Davis Institute-Jewish General Hospital,

Montréal, Quebec, Canada H3T 1E2

e-mail: chen.liang@mcgill.ca

B. D. Kuhl $\cdot$ M. A. Wainberg $\cdot$ C. Liang

Department of Medicine, McGill University,

Montréal, Quebec, Canada H3A 2B4

V. Cheng $\cdot$ M. A. Wainberg $\cdot$ C. Liang

Department of Microbiology and Immunology, McGill University,

Montréal, Quebec, Canada H3A 2B4 by cellular pathogen recognition receptors that are located on cellular membranes or within the cytosol (reviewed in Kawai and Akira 2006; Douville and Hiscott 2010). For example, membrane-associated Toll-like receptor 3 (TLR3) recognizes double-stranded viral RNA, recruits TIR domain-containing adaptor inducing IFN- $\beta$, and activates the interferon regulatory factor 3 (IRF3) and nuclear factor kappa-light-chain-enhancer of activated B cells (NF-kB) pathways to induce type I interferon production. TLR7 and TLR9 are both located in endosomes. After recognizing single-stranded viral RNA or double-stranded viral DNA, they recruit myeloid differentiation primary response gene 88 and activate the IRF7 and NF-KB pathways. The cytosolic retinoic-acid-inducible gene I and melanoma differentiation-associated gene 5 recognize either doublestranded viral RNA or 5'-triphospho viral RNA and activate the MAVS (also known as IPS-1, VISA, and Cardif) signaling transduction pathway, which leads to interferon production.

Interferon binds to its receptor on the cell surface and activates tyrosine kinase 2 and Janus kinase 1 that in turn phosphorylates signal transducer and activator of transcription 1 (STAT1) and STAT2. The phosphorylated STAT1/ STAT2 then translocates into the nucleus and forms the interferon-stimulated gene factor 3 (ISGF3) complex together with IRF9. The ISGF3 complex activates the expression of a wide spectrum of genes, collectively named interferon-stimulated genes (ISGs) that together establish an antiviral state. Among hundreds of ISGs, those with welldefined antiviral activities include RNaseL/OAS (2', $5^{\prime}$ oligoadenylate synthetase), ISG15, myxovirus resistance $(\mathrm{Mx})$, protein kinase $\mathrm{R}$ (PKR), and adenosine deaminase, RNA-specific (ADAR) that inhibit virus infection by different mechanisms (reviewed in Sadler and Williams 2008). Upon binding to double-stranded RNA, OAS 
oligomerizes and synthesizes $2^{\prime}, 5^{\prime}$-oligoadenylates that bind to and activate RNaseL. The activated RNaseL degrades both cellular and viral RNA. ISG15 is conjugated to cellular and viral proteins to modulate cellular response and inhibit virus infection. This event is termed ISGylation that is catalyzed sequentially by three enzymes called ubiquitin E1 enzyme, E2-conjugating enzymes (such as UBCH8), and E3 ligase enzymes (such as HERC5, homologous to the E6-associated $\mathrm{C}$ terminus domain and RCC1-like-domain-containing protein 5). Mx proteins are dynamin-like large GTPases. They inhibit the infection of a wide range of RNA and DNA viruses by binding to viral nucleocapsids or other viral components and subsequently trapping viral complexes as a result of $\mathrm{Mx}$ oligomerization. PKR is a kinase that is dormant in its monomeric form. Upon binding to viral double-stranded RNA, the kinase activity is activated, which leads to phosphorylation and dimerization of PKR. The activated PKR then phosphorylates EIF $2 \alpha$ and thus inhibits translation of cellular and viral proteins. ADAR is an adenosine deaminase that binds double-stranded RNA and converts adenosine to inosine. In addition to these antiviral effectors, recent studies discovered that an ISG named tetherin (also called BST-2/CD317/ HM1.24, bone marrow stromal cell antigen 2) inhibits viral infection via a novel mechanism by blocking the release of nascent enveloped viral particles from the cell surface.

\section{Discovery of tetherin as an antiviral protein}

BST-2 (or HM1.24) was first reported as a protein that expresses at the surface of human plasma cell lines, bone marrow stromal cell lines and B cells, and is thus speculated to have a role in B-cell development (Goto et al. 1994; Ishikawa et al. 1995). Its potential role as a component of the host antiviral defense mechanism was suggested by its being a target of the $\mathrm{K} 5$ protein (also named MIR2) of Kaposi's sarcoma-associated herpesvirus (Bartee et al. 2006). The antiviral activity of BST-2 was subsequently demonstrated for its ability to inhibit the release of HIV-1 that is deficient in Vpu and was therefore given the name tetherin (Neil et al. 2008; Van Damme et al. 2008).

In addition to structural and enzymatic proteins including Gag, Pol, and Env, HIV-1 also encodes two regulatory proteins Tat and Rev that are essential for viral gene expression, as well as four accessory proteins named $\mathrm{Vpr}$, Vif, Vpu, and Nef that play important roles in HIV-1 pathogenesis (reviewed in Anderson and Hope 2004; Malim and Emerman 2008). For example, Vif recognizes a cellular cytidine deaminase named apolipoprotein $\mathrm{B}$ mRNA editing enzyme, catalytic polypeptide $3 \mathrm{G}$ (APOBEC $3 \mathrm{G}$ ), triggers polyubiquitination of $\mathrm{APOBEC} 3 \mathrm{G}$ through recruiting the
Cullin5-containing E3 ligase, and sends APOBEC3G to proteasomes for degradation. In the absence of Vif, APOBEC 3G associates with HIV-1 RNP complex, gets packaged into virus particles, and induces hypermutation of viral cDNA by converting cytidines into uridines during reverse transcription (reviewed in Henriet et al. 2009; Niewiadomska and Yu 2009). It has been known for quite a long time that $\mathrm{Vpu}$ is required for efficient HIV-1 production in certain cell lines such as HeLa and primary T cells but not in cells such as HEK293 and COS-7 (Strebel et al. 1989; Göttlinger et al. 1993). The Vpu-deficient HIV1 particles encounter a difficulty of leaving the surface of Vpu-non-permissive cells such as HeLa. A dominant cellular factor was speculated to underlie this restriction activity because the restriction phenotype was recreated in the $\mathrm{HeLa} / \mathrm{COS}-7$ heterokaryotic cells (Varthakavi et al. 2003). Subsequent studies revealed that this putative restriction factor is interferon-inducible (Neil et al. 2007). A long quest for this mysterious factor by several labs led to the discovery of its identity as tetherin, named after its function to tether nascent HIV-1 particles to the cell surface (Neil et al. 2008; Van Damme et al. 2008). After this finding, a variety of enveloped viruses were also shown to be subject to the restriction of tetherin. These include HIV-2 (Le Tortorec and Neil 2009), simian immunodeficiency virus (SIV) (Jouvenet et al. 2009), alpharetrovirus (Rous sarcoma virus) (Jouvenet et al. 2009), betaretrovirus (Mason-Pfizer monkey virus) (Jouvenet et al. 2009), gammaretrovirus (human T-cell leukemia virus type 1 (HTLV-1)) (Jouvenet et al. 2009), filoviruses (Marburg virus (Jouvenet et al. 2009; Sakuma et al. 2009) and Ebola virus (Jouvenet et al. 2009; Kaletsky et al. 2009)), arenaviruses (Lassa virus (Sakuma et al. 2009)), and herpesvirus (Kaposi's sarcoma-associated herpesvirus (KSHV) (Mansouri et al. 2009)). The two forthcoming questions are how tetherin retains enveloped viral particles to the cell surface and how viruses evade this restriction.

\section{What is tetherin?}

Human tetherin is encoded by a single copy of the bst-2 gene on chromosome 19p13.2 that is split into four exons (Ishikawa et al. 1995). This gene is relatively nonpolymorphic with only one nonsynonymous singlenucleotide polymorphism that is listed in the human genome database and located in the coding region for the ectodomain of tetherin. Tetherin orthologs are present in the genomes of mammals but with significant sequence divergence (McNatt et al. 2009).

Tetherin is constitutively expressed in mature B cells, some cancer cell lines, bone marrow stromal cells, monocyte-derived macrophages, and plasmacytoid dendrit- 
ic cells (pDCs) (Ishikawa et al. 1995; Vidal-Laliena et al. 2005; Blasius et al. 2006; Mitchell et al. 2009; Miyagi et al. 2009). Interferon strongly stimulates tetherin expression due to the presence of interferon response elements and a binding site for STAT3 in the promoter region of bst-2 gene (Blasius et al. 2006). However, the level of tetherin expression as part of the interferon response appears to be cell-type-dependent (Miyagi et al. 2009). Regarding cancer T-cell lines, which are commonly used in laboratories to study HIV infection, the basal expression levels of tetherin vary but are all interferon-inducible (Neil et al. 2008; Van Damme et al. 2008; Rong et al. 2009).

When first described in B cells, tetherin was suggested to be a marker for B-cell maturation (Ishikawa et al. 1995). Its subsequent detection on multiple myeloma cells suggested a tetherin-based therapeutic strategy to induce cell-mediated cytotoxicity with tetherin antibody (Ozaki et al. 1997; Ono et al. 1999). In an epithelial cell line, tetherin interacts and modulates the subapical actin cytoskeleton, indirectly, via RICH2, EBP50, and ezrin (Rollason et al. 2009). It remains undetermined whether the cytoskeleton-modulating activity is involved in tetherin's function of restricting viral release. Tetherin is also a ligand for immunoglobulin-like transcript 7 (ILT7) on pDCs (Cao et al. 2009). Since ILT7-mediated signaling strongly inhibits production of interferon and other cytokines in pDCs (Brown et al. 2004; Cao et al. 2006), tetherin may negatively regulate interferon and cytokine production (Cao et al. 2009).

Tetherin localizes to the plasma membrane, the trans-Golgi network (TGN) and the early and recycling endosomes, and cycles between these membrane compartments (Kupzig et al. 2003; Habermann et al. 2010). Endocytosis of tetherin from the plasma membrane is clathrin-dependent and involves an interaction of tetherin with the adaptor proteins AP-1 and AP-2 (Rollason et al. 2007; Masuyama et al. 2009). In an epithelial cell line, tetherin localizes to the apical membrane following cellular polarization (Rollason et al. 2009). At the cell surface, tetherin localizes to lipid rafts (Kupzig et al. 2003; Rollason et al. 2007, 2009; Goffinet et al. 2009). Immunofluorescence microscopy reveals tetherin as punctual clusters at the cell surface (Hinz et al. 2010). HIV-1 Gag assembly and virus budding also occur at these membrane microdomains (Waheed and Freed 2009). In addition, lipid rafts are also involved in forming virological synapses and promoting cell-to-cell transmission of viruses (Popik et al. 2002; Piguet and Sattentau 2004; Popik and Alce 2004; Jolly and Sattentau 2005).

\section{How does tetherin block virus release?}

Tetherin retains nascent viral particles at the cell surface by linking them to the cellular membrane and to each other
(Fig. 1a). Electron microscopy shows accumulation of viral particles at the surface of tetherin-expressing cells (Neil et al. 2008; Perez-Caballero et al. 2009; Fitzpatrick et al. 2010). Tetherin is colocalized with viral Gag at the cell surface (Neil et al. 2008; Van Damme et al. 2008; PerezCaballero et al. 2009) and is detected in the membrane of viruses that are released from the cell surface either spontaneously (Fitzpatrick et al. 2010; Habermann et al. 2010) or by shearing force (such as vortexing) (Miyagi et al. 2009). Results of immunoelectron microscopy further revealed that tetherin is located between the tethered viral particles and between the tethered virus particle and the plasma membrane, which indicates a direct role of tetherin in blocking virus release. This conclusion is further supported by two lines of evidence. First, human tetherin, when expressed in non-human cells, can restrict release of budding virions (Sato et al. 2009). Second and more importantly, the antiviral function of tetherin is, to a certain degree, independent of its sequence. An antiviral competent artificial tetherin has been reported, which has its transmembrane domain derived from transferrin receptor, coiledcoil domain from dystrophia myotonica protein kinase, and glycophosphoinisitol (GPI) anchor from urokinase plasminogen activator receptor (Perez-Caballero et al. 2009). These results suggest that the antiviral activity of tetherin unlikely requires a co-factor.

Structural determinants of tetherin's antiviral function Tetherin is a type II transmembrane protein (Ishikawa et al. 1995; Kupzig et al. 2003). It bears a short cytoplasmic Nterminal region, a transmembrane region, an ectodomain, and a C-terminal GPI anchor (Kupzig et al. 2003). This topology is rare and only shared with an isoform of the prion protein (Hegde et al. 1998; Stewart et al. 2001). Tetherin forms disulfide-linked dimers. It is heterogeneously glycosylated and thus migrates as 30-36-kDa proteins on a sodium dodecyl sulfate-polyacrylamide gel. These structural features together determine tetherin's antiviral function and also regulate its sensitivity to different viral antagonists.

The short cytoplasmic domain (at amino acid positions 1 to 21) contains a conserved dual tyrosine motif $\left(\mathrm{YXY}_{6-8}\right)$ that is crucial for clathrin-mediated endocytosis through recruiting AP-1 and AP-2 adaptor proteins (Rollason et al. 2007). The two lysine residues (at positions 18 and 21) are ubiquitinized by the RING-CH K5 ligase of KSHV, which leads to tetherin degradation (Mansouri et al. 2009; Pardieu et al. 2010). Human tetherin lacks the 14-D/GDIWK-18 motif that constitutes the interaction site of Nef and is thus resistant to Nef-mediated antagonism (Jia et al. 2009; Zhang et al. 2009). Additionally, the cytoplasmic domain of tetherin has been described to indirectly modulate subapical actin cytoskeleton in polarized endothelial cells via RICH2, EBP50, and ezrin (Rollason et al. 2009). 
Fig. 1 Antiviral mechanism of tetherin. a Tetherin localizes to the virus budding site and incorporates into the viral membrane. Tetherin exerts its antiviral action by tethering viral particles to the cell and to each other. Further, tetherin inhibits protease-mediated viral maturation of released viral particles. b, c Models of molecular tethering mechanism. Tetherin homodimers are likely oriented in parallel. Tetherin links viral particles to cells by insertion of one terminus into the virion membrane, while the other is located in the cellular membrane (b), or tetherin may link viral particles and cells via dimerization of the ectodomains of monomers with both termini inserted in either the virion membrane or the cellular membrane

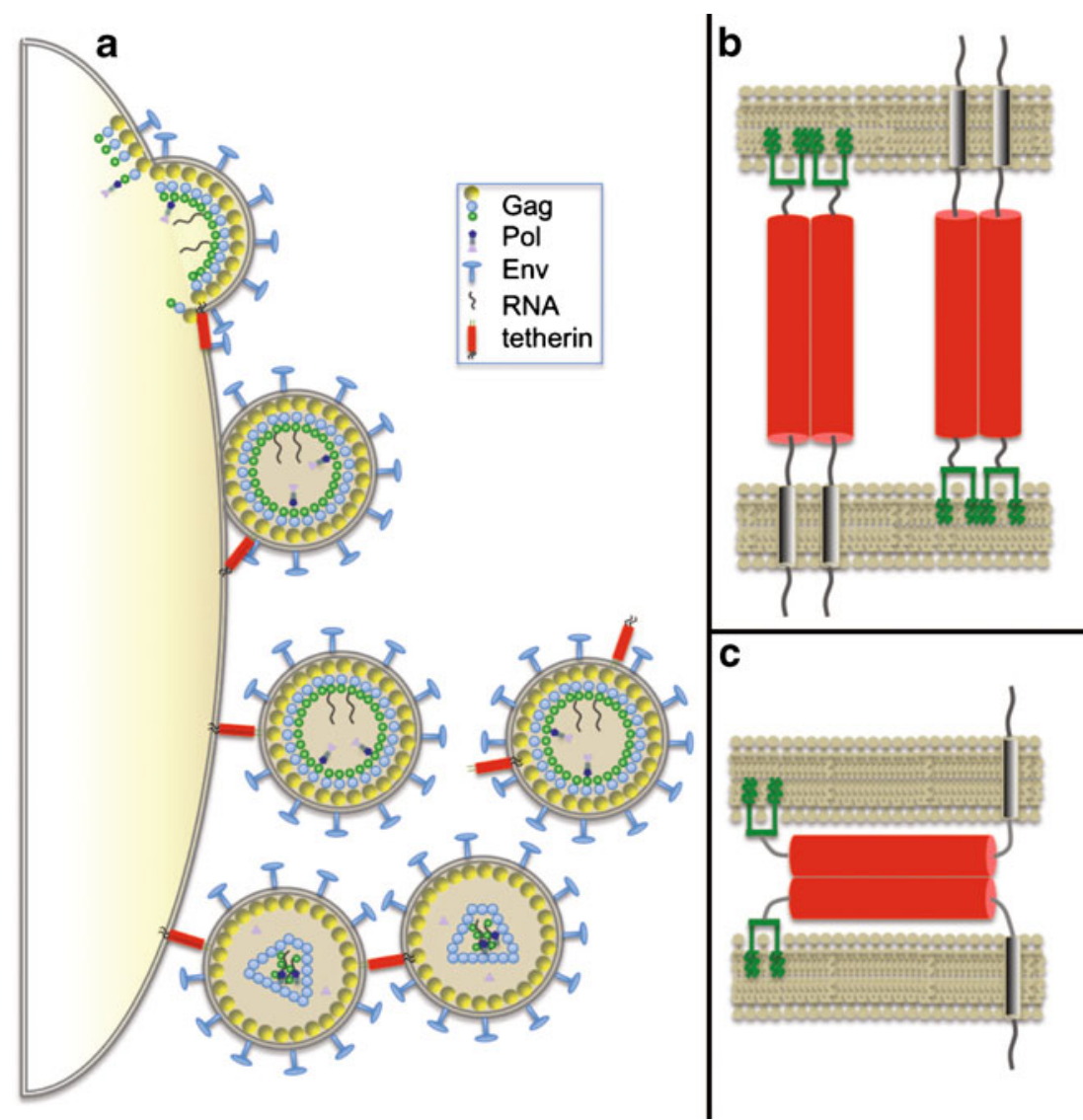

The transmembrane region (at amino acid positions 22 to 43 ) consists of a single membrane-spanning helix. In the case of HIV-1, the transmembrane region renders human tetherin sensitive to Vpu-mediated downmodulation from the cell surface and subsequent degradation (Gupta et al. 2009a; McNatt et al. 2009; Rong et al. 2009). Several amino acid residues across the transmembrane domain of tetherin have been reported to regulate the sensitivity to $\mathrm{Vpu}$ and tetherin/Vpu interaction; these include L22, L23, G25, I26, V30, I33, I36, and T45 (Fig. 2) (Gupta et al. 2009a; McNatt et al. 2009; Rong et al. 2009). Interestingly, results of computer modeling of the structure of tetherin transmembrane domain show that these amino acid residues are positioned at one side of the helix, suggesting that they may together form the contact site with Vpu (Fig. 2).

The ectodomain (amino acids 44 to 160) assumes a long single $\alpha$-helix as shown by the results of X-ray crystallography (Fig. 3) (Hinz et al. 2010; Schubert et al. 2010; Yang et al. 2010a). The complete ectodomain extends to a length of 150 to $170 \AA$ which includes the 90 - $\AA$ C-terminal coiledcoil domain (Hinz et al. 2010). Two tetherin molecules dimerize via this parallel disulfide-linked coiled-coil structure that is mainly stabilized by interactions throughout the two-third C-terminal portion of the ectodomain (Fig. 3). The N-terminal portion of ectodomain appears to be relatively flexible at two hinges (positions A88 and G109) and mediates the tetramerization of two tetherin dimers by forming an antiparallel four-helix bundle (Fig. 3) (Hinz et al. 2010; Schubert et al. 2010). Although two tetherin dimers form a tetramer in crystals, mutants that are deficient in tetramerization retain most of the antiviral activity (Schubert et al. 2010; Yang et al. 2010a). The length of ectodomain is crucial for tetherin to block virus release, which suggests a molecular ruler function to keep the two membrane-spanning termini at a distance that is required for maximal antiviral activity (Hinz et al. 2010; Yang et al. 2010a).

The ectodomain of tetherin also contains three cysteines $\left(\mathrm{C}_{53} \mathrm{C}_{63} \mathrm{C}_{91}\right)$ that form disulfide bonds and therefore stabilize tetherin homodimers. In addition, there exist two sites for post-translational N-linked glycosylation $\left(\mathrm{N}_{65} \mathrm{~N}_{92}\right)$ (Ohtomo et al. 1999; Andrew et al. 2009). While glycosylation was shown to be dispensable for the antiviral function, formation of at least one disulfide bond is crucial for tetherin to block virus release (Andrew et al. 2009; Perez-Caballero et al. 2009; Hinz et al. 2010).

The C-terminal region of tetherin contains a signal for the addition of a GPI anchor. The nascent protein is predictably cleaved at a serine residue (position 160) in the endoplasmic reticulum (ER) followed by the attachment 

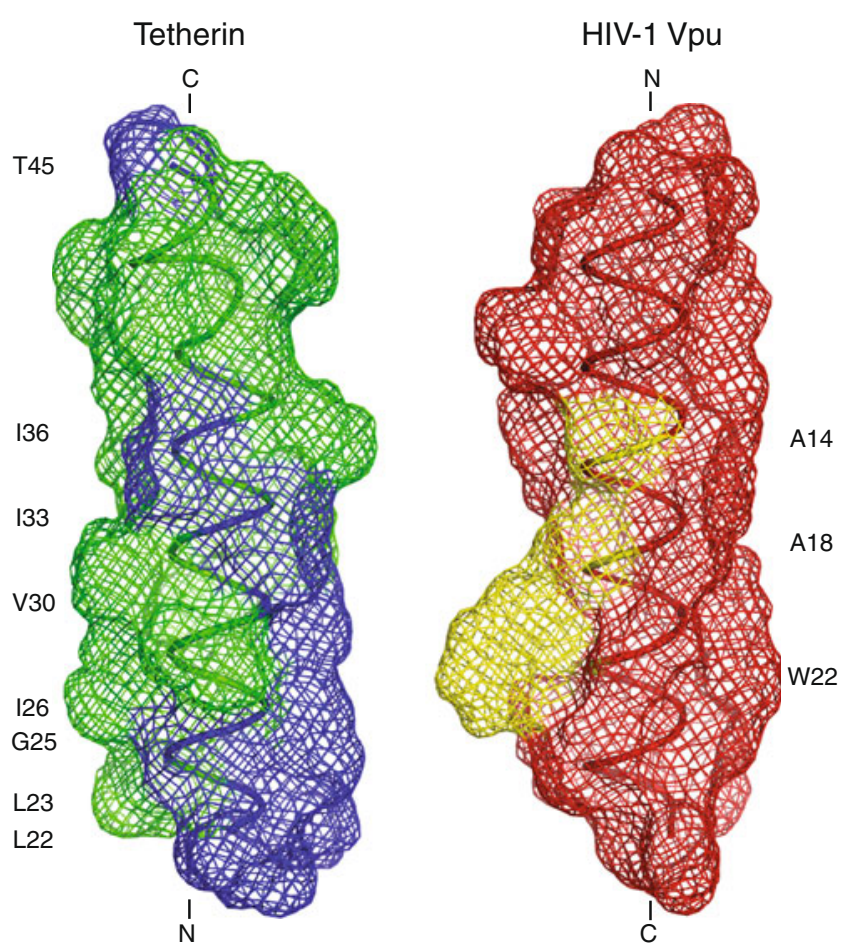

Fig. 2 Models of tetherin and Vpu transmembrane domains. The transmembrane domains of human tetherin (left) and HIV-1 Vpu (right) are illustrated in regard to their orientation in the membrane. Amino acids of the tetherin transmembrane known to impact on the interaction without abruption of tetherin function are highlighted in blue and indicated left to the model; amino acids are selected according to Gupta et al. (2009a), McNatt et al. (2009), and Rong et al. (2009). Amino acids that are involved in tetherin downmodulation from the cell surface are highlighted in yellow on the Vpu transmembrane model (Vigan and Neil 2010). Models were created with PyMol software on the basis of the sequences of human tetherin (GenBank ID NP_004326.1) and HIV-1 Vpu from viral clone pNL_4-3 (GenBank ID AAK08488.1)

of the prefabricated GPI anchor (Kupzig et al. 2003). Therefore, the mature tetherin is a heterogeneously glycosylated 160-amino-acid single pass transmembrane protein with a C-terminal GPI anchor incorporated into lipid rafts. The GPI anchor-mediated localization to lipid rafts promotes the clathrin-mediated endocytosis (Rollason et al. 2007). Removal of GPI anchor abolishes the antiviral activity of tetherin (Neil et al. 2008; Perez-Caballero et al. 2009).

Models of "tethering" Several models have been proposed to illustrate how tetherin retains viral particles on the cell surface. These models are conceived on the basis of two key features of tetherin, dimerization across the ectodomain and insertion of the two termini into either the cellular plasma membrane or the membrane of viral particle. Since not only does tetherin act as a link to retain a virus particle to the cell surface but also tethers the viral particles to each other, it is believed that the $\mathrm{N}$ and the $\mathrm{C}$ termini of tetherin do not distinguish between the cellular and the viral membranes for insertion, which is not surprising given that viral membrane is derived from the cellular plasma membrane (Fig. 1b, c) (Neil et al. 2008; Perez-Caballero et al. 2009). Theoretically, a tetherin dimer may consist of two monomers that are positioned either in a parallel or in an antiparallel orientation. These two possibilities are now resolved by the crystal structures of tetherin ectodomains that are shown to form parallel dimers. Tetherin may thus function as a parallel homodimer to bridge cellular and viral membrane directly by insertion of one terminus into the cellular membrane and the other into the viral membrane (Fig. 1b). However, there is no conclusive evidence to rule out the possibility that tetherin dimers work by inserting the two termini of the same tetherin molecule into the same membrane (Fig. 1c).

Fate of tethered viral particles The tethered virions may be internalized and sent to late endosomes and lysosomes for degradation (Neil et al. 2007; Van Damme et al. 2008; Sato et al. 2009). It was recently shown that internalization and lysosomal degradation are promoted by the interaction of the cytosolic domain of tetherin with breast cancer associated gene 2 (Rabring7, ZNF364, RNF115) (Miyakawa et al. 2009). However, the molecular mechanisms behind this remain unknown. Furthermore, it is unclear whether internalized virions can cycle back to the cell surface if not degraded.
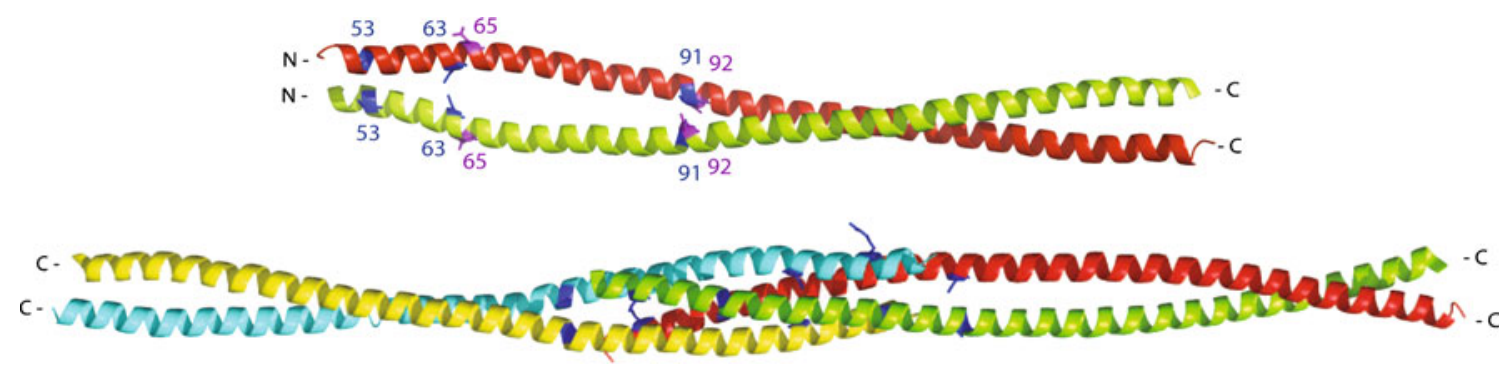

Fig. 3 Crystal structure of tetherin ectodomain. Shown are the crystal structures of a tetherin dimer (top) and tetramer (bottom). The disulfide bond building cysteine residues $\mathrm{C}_{53} \mathrm{C}_{63} \mathrm{C}_{91}$ are highlighted in blue (top and bottom); N-glycosylation sites $\mathrm{N}_{65} \mathrm{~N}_{92}$ are presented in purple (top). Structures are based on the X-ray crystallography data from Schubert et al. (2010) (PDB ID: 3NWH) and were created using PyMol software. Structures of the ectodomains were created using PyMol software 
By tethering the nascent virus to the cell surface, tetherin may affect cell-to-cell virus transmission. Cell-to-cell transmission takes place through the intercellular contact zones, termed virological synapses that temporarily connect the infected cell to the uninfected one (reviewed in Piguet and Sattentau 2004; Sattentau 2008; Martin and Sattentau 2009). Studies have been performed to assess the effect of tetherin on cell-to-cell transmission of HTLV-1 and HIV-1. Results showed that the tethered HTLV-1 particles on the cell surface promote direct cell-to-cell spreading of HTLV-1 (Pais-Correia et al. 2009). In the case of HIV-1, two studies reported a decreased cell-to-cell transmission as a result of the presence of tetherin at virological synapse (Casartelli et al. 2010; Kuhl et al. 2010b), whereas a third study observed the opposite effect (Jolly et al. 2010). Furthermore, differences were reported regarding the impact of tetherin on the infectivity of the transferred HIV-1 particles (Casartelli et al. 2010; Jolly et al. 2010; Kuhl et al. 2010b). Since cell-tocell transmission is the predominant mode of HIV-1 spreading in cell culture and in secondary lymphoid tissues, clarification of the role of tetherin in cell-to-cell dissemination of HIV-1 will be of great importance.

In addition to holding viral particles to the cell surface, tetherin impairs the infectivity of Vpu-deficient HIV-1 particles that are released into the culture supernatant. Several groups have observed that tetherin reduces the levels of infectious Vpu-deficient HIV-1 particles to a more severe degree rather than decreasing the total amounts of virus particles as measured by viral $\mathrm{CA}(\mathrm{p} 24)$ or viral reverse transcriptase activity, which implicates an adverse effect of tetherin on the infectivity of progeny virions (Neil et al. 2008; Van Damme et al. 2008; Perez-Caballero et al. 2009; Sauter et al. 2009). Further studies revealed a defect in the morphology of cell-free HIV-1 particles that may result from incomplete processing of viral Gag proteins (Zhang and Liang 2010). The mechanism of this interference, however, is not yet clear.

\section{How do viruses evade tetherin restriction?}

Viruses have evolved different strategies to evade tetherin restriction. One common strategy that some viruses share is to shut down the interferon pathway such that tetherin is not induced for expression upon viral infection (reviewed in Versteeg and García-Sastre 2010). With this mechanism, not only tetherin but also other interferon-stimulated antiviral factors are not expressed in virus-infected cells. On the other hand, some viruses encode proteins that act as antagonists to counteract tetherin. The known tetherin antagonists include $\mathrm{Vpu}$ of HIV-1, envelope protein of HIV-2, SIVtan and Ebola virus, Nef of SIV, and K5 of KSHV. These viral proteins overcome tetherin restriction by different mechanisms (summarized in Fig. 4 and Table 1).

$H I V-1 \quad V p u$ HIV-1 Vpu is the most extensively studied tetherin antagonist among the others. $\mathrm{Vpu}$ is expressed from the vpu-env bi-cistronic viral RNA as a $16-\mathrm{kDa}$ protein (reviewed in Bour and Strebel 2003; Malim and Emerman 2008). Although it is translated at the late stage of HIV-1 infection, $\mathrm{Vpu}$ is not found in virions. There exists a 27-amino-acid transmembrane domain at the $\mathrm{N}$ terminus of $\mathrm{Vpu}$, followed by a 54-amino-acid cytoplasmic domain that consists of two $\alpha$-helices (the number of amino acids refers to Vpu of the NL4-3 strain). It is worth noting that there are two serine residues at positions 52 and 56 that are located in the linker region of these two helices and are subject to phosphorylation. It is well documented that Vpu downregulates CD4 at the ER and thus prevents premature binding of viral gp160 with CD4. This function of Vpu initiates with recognition of the cytoplasmic tail of CD4 and recruitment of $\beta-\operatorname{TrCP}$ (a human beta transducin repeat containing protein) that is a key component of the E3 ubiquitin ligase complex (Skp1, Cullin1, and $\beta$-TrCP). The phosphorylated S52 and S56 residues of Vpu constitute the binding site for $\beta$-TrCP that subsequently causes polyubiquitination and proteasomal degradation of CD4.

The second prominent function of $\mathrm{Vpu}$ is to promote HIV-1 release by counteracting tetherin (Neil et al. 2008; Van Damme et al. 2008). One mechanism underlying this function of $\mathrm{Vpu}$ is to downregulate cell surface expression of tetherin and therefore removes tetherin from HIV-1 assembly and budding site on the plasma membrane (Van Damme et al. 2008; Perez-Caballero et al. 2009). The existence of additional mechanisms is also suggested by the observation of a significant level of tetherin incorporation into wild-type HIV-1 particles (Habermann et al. 2010). More importantly, $\mathrm{Vpu}$ enhances virus production in T-cell lines such as CEMx174 and $\mathrm{H} 9$ cells without reducing cell surface expression of tetherin (Miyagi et al. 2009).

Vpu may achieve tetherin downregulation from the cell surface by two mechanisms, depletion of the intracellular tetherin pool and sequestration of tetherin at the TGN. Vpu that is either produced from an expression vector or from HIV-1 proviral DNA is capable of diminishing the total amounts of tetherin in cells. This activity of Vpu relies on the recruitment of $\beta$-TrCP (Douglas et al. 2009; Mangeat et al. 2009; Mitchell et al. 2009). Mutating the S52/S56 residues of $\mathrm{Vpu}$ or depleting $\beta$-TrCP with small interfering RNA oligos significantly attenuates Vpu's antitetherin activity. In addition, expression of a dominant negative mutant of $\beta-\operatorname{TrCP}$ such as $\beta-\operatorname{TrCP}-\Delta \mathrm{F}$ also cripples the ability of $\mathrm{Vpu}$ to counteract tetherin. However, mutation of the only two lysine residues, the most common ubiquitin receptor, within the cytoplasmic tail of tetherin fails to 


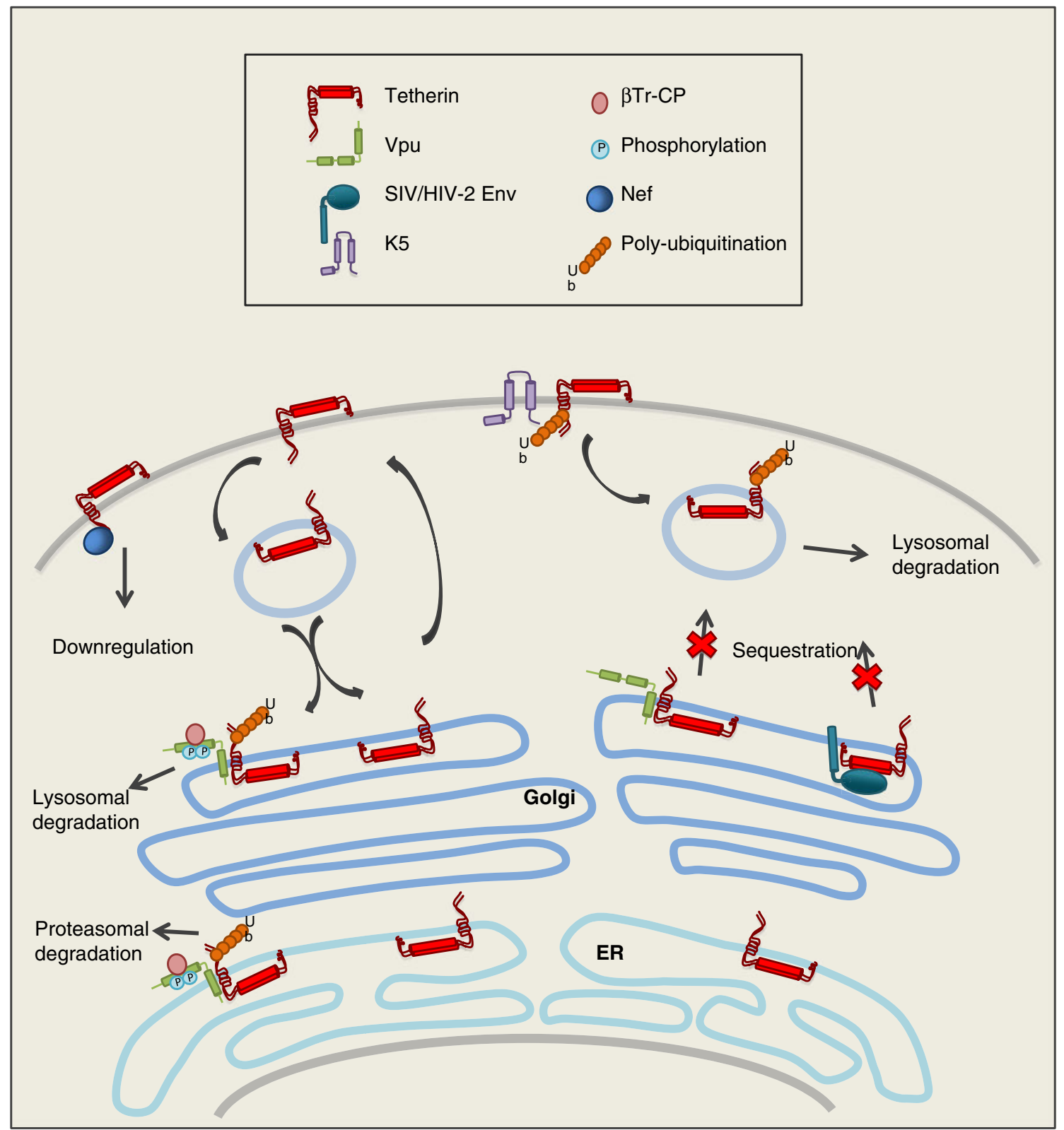

Fig. 4 Illustration of viral counter measures against tetherin. The normal cellular trafficking of tetherin is depicted from the endoplasmic reticulum $(E R)$ to the Golgi apparatus, to the plasma membrane, and then through recycling endosomes. This trafficking is interrupted by several viral proteins that have been identified to antagonize tetherin. Vpu may sequester tetherin at the TGN or recruit $\beta$-TrCP to

render the mutated tetherin resistant to Vpu-mediated degradation. The major Vpu-responsive site has now been mapped to the STS motif (positions 3 to 5 ) that undergoes $\mathrm{Vpu} / \beta$-TrCP-dependent ubiquitination (Tokarev et al. 2010).

Vpu-mediated tetherin degradation may require either the proteasomal or the endosomal/lysosomal pathway. Interestingly, blocking either of these two degradation pathways by specific inhibitors in different cell lines ubiquitinate tetherin and target it for degradation via the lysosomal or proteasomal pathway. Nef interacts with the cytoplasmic tail of tetherin and triggers tetherin downregulation from the cell surface. SIV/HIV-2/Ebola envelope proteins may sequester tetherin at the TGN/perinuclear regions. K5 ubiquitinates tetherin and causes tetherin degradation in lysosomes

rescues Vpu-mediated tetherin degradation, albeit the extent of rescue varies due to specific experimental settings. Concanamycin A (ConA) is an inhibitor of the endosomal/lysosomal pathway and was observed to stabilize tetherin in the presence of $\mathrm{Vpu}$ (Douglas et al. 2009). Bafilomycin A1 (BafA1) reverses the cell surface and intracellular downregulation of tetherin by Vpu (Mitchell et al. 2009). Both BafAl and ConA inhibit the endosomal vacuolar ATPase and prevent acidification of endosome and 
Table 1 Summary of viral tetherin antagonists

\begin{tabular}{|c|c|c|c|c|c|c|}
\hline $\begin{array}{l}\text { Tetherin } \\
\text { antagonists }\end{array}$ & $\begin{array}{l}\text { Tetherin } \\
\text { surface } \\
\text { downregulation }\end{array}$ & $\begin{array}{l}\text { Tetherin } \\
\text { intracellular } \\
\text { depletion }\end{array}$ & $\begin{array}{l}\text { Target domain } \\
\text { in tetherin }\end{array}$ & $\begin{array}{l}\text { Intracellular } \\
\text { relocalization } \\
\text { of tetherin }\end{array}$ & $\begin{array}{l}\text { Species } \\
\text { specificity }\end{array}$ & References \\
\hline HIV-1 Vpu & Yes & Yes & TM domain & TGN & Yes & $\begin{array}{l}\text { Neil et al. (2008), Van } \\
\text { Damme et al. (2008), } \\
\text { Mitchell et al. (2009), } \\
\text { Goffinet et al. (2009), } \\
\text { Douglas et al. (2009), } \\
\text { Mangeat et al. (2009), } \\
\text { Iwabu et al. (2009), } \\
\text { Dubé et al. (2010) }\end{array}$ \\
\hline SIVcpz/gor Vpu & $?$ & $?$ & $?$ & $?$ & Yes & $\begin{array}{l}\text { Sauter et al. (2009), } \\
\text { Yang et al. (2010b) }\end{array}$ \\
\hline SIVgsn/mus/mon Nef & $?$ & $?$ & $\begin{array}{l}\text { Cytoplasmic tail } \\
\text { (G/DDIWK) }\end{array}$ & $?$ & Yes & $\begin{array}{l}\text { Sauter et al. (2009), } \\
\text { Yang et al. (2010b) }\end{array}$ \\
\hline SIVmac/smm Nef & Yes & $?$ & $\begin{array}{l}\text { Cytoplasmic tail } \\
\text { (GDDIWK) }\end{array}$ & $?$ & Yes & $\begin{array}{l}\text { Jia et al. (2009), } \\
\text { Zhang et al. (2009) }\end{array}$ \\
\hline SIVagmSab/Tan Nef & $?$ & $?$ & $\begin{array}{l}\text { Cytoplasmic tail } \\
\text { (DDICK) }\end{array}$ & $?$ & Yes & Zhang et al. (2009) \\
\hline HIV-2 Env & Yes & No & Ectodomain & TGN & No & $\begin{array}{l}\text { Le Tortorec and Neil (2009), } \\
\text { Jia et al. (2009), } \\
\text { Hauser et al. (2010), } \\
\text { Abada et al. (2005) }\end{array}$ \\
\hline Ebola GP & No & No & $?$ & $?$ & No & $\begin{array}{c}\text { Kaletsky et al. (2009), } \\
\text { Lopez et al. (2010) }\end{array}$ \\
\hline SIVtan Env & Yes & No & Ectodomain & Perinuclear region & No & Gupta et al. (2009b) \\
\hline KSHV K5 & Yes & Yes & Cytoplasmic tail(K18) & Late endosomes & Yes & $\begin{array}{l}\text { Mansouri et al. (2009), } \\
\text { Pardieu et al. (2010) }\end{array}$ \\
\hline
\end{tabular}

its maturation to late endosome. A study using a lysosomal protease inhibitor cocktail of leupeptin, pepstatin $\mathrm{A}$, and E64d also supports the role of endosomal/lysosomal pathway in Vpu-mediated degradation of tetherin (Iwabu et al. 2009). Finally, knockdown of the $\mu 2$ subunit of the AP-2 adaptor complex partially prevents the Vpu-induced tetherin downregulation (Mitchell et al. 2009).

MG-132 has been used in several studies as a proteasome inhibitor to prevent tetherin degradation by $\mathrm{Vpu}$, but the results showed varying degrees of efficacy (Van Damme et al. 2008; Goffinet et al. 2009; Mangeat et al. 2009; Mitchell et al. 2009). It is noted that long-term treatment with MG-132 may deplete the intracellular pool of free ubiquitin and thus affect the ubiquitin-dependent degradation by either the proteasomes or the lysosomes (Van Damme et al. 2008; Douglas et al. 2009; Mitchell et al. 2009). Given the important role of $\beta$-TrCP and ubiquitination in the degradation of tetherin by $\mathrm{Vpu}$, results obtained with MG-132 treatment alone may not be sufficient to distinguish between the roles of proteasomal or endolysosomal pathway in tetherin degradation. The use of the other proteasome inhibitors ALLN and clastolactacystin $\beta$-lactone (inhibitor of the $20 \mathrm{~S}$ proteasome) increases the level of intracellular tetherin in the presence or the absence of Vpu (Goffinet et al. 2009). Notably, both proteasome inhibitors abolish the ability of $\mathrm{Vpu}$ to rescue viral release in the presence of tetherin (Goffinet et al. 2009). It is possible that both the proteasomal and the endolysosomal pathways are involved in Vpu-mediated degradation of tetherin, albeit likely to varying degrees in different cell lines.

In addition to degradation, $\mathrm{Vpu}$ may disturb tetherin trafficking in cells and sequester tetherin away from the plasma membrane. Tetherin is normally localized to the plasma membrane and to, a less degree, in the perinuclear regions. Vpu causes a relocalization of tetherin from the plasma membrane to the TGN where tetherin is sequestered (Dubé et al. 2010). Results of immunoelectron microscopy showed an increased presence of tetherin at the early/ recycling endosomes when $\mathrm{Vpu}$ is expressed (Habermann et al. 2010). It is conceivable that Vpu interacts with tetherin and diverts the trafficking of tetherin toward specific intracellular membrane compartments for sequestration or degradation.

$\mathrm{Vpu}$ likely recognizes tetherin through an interaction between the transmembrane domains of these two proteins. This scenario was first suggested by the finding that HIV-1 $\mathrm{Vpu}$ is unable to counteract tetherin from monkeys such as 
African green monkey and Rhesus monkeys (Gupta et al. 2009a; McNatt et al. 2009; Rong et al. 2009). This resistance phenotype of monkey tetherin to HIV-1 Vpu was subsequently mapped to a few amino acid residues in the transmembrane domain of tetherin, which are divergent from their counterparts in the human tetherin transmembrane domain (Gupta et al. 2009a; Jia et al. 2009; McNatt et al. 2009; Zhang et al. 2009). A detailed mutagenesis analysis of the $\mathrm{Vpu}$ transmembrane domain also identified several residues that determine Vpu's tetherin antagonism activity (Vigan and Neil 2010). The Vpu mutants A14L, W22A, A14L/W22A, and, to a minor extent, A18L lost their ability to counteract tetherin (Vigan and Neil 2010). Further experiments showed that these Vpu mutants were crippled in downregulating tetherin from cell surface and were impaired in binding to tetherin. It is worth noting that the W22A mutant is also defective in downregulating CD4 from cell surface (Vigan and Neil 2010). In solid-state NMR structures of Vpu transmembrane multimers, A14, A18, and W22 line up on the outside-facing side of the helices (Park et al. 2003; Vigan and Neil 2010). These amino acid residues are likely involved in forming the interaction interface between the $\mathrm{Vpu}$ and the tetherin transmembrane helices (Fig. 2). In support of their importance in Vpu function, A18 and W22 are highly conserved in HIV-1 isolates that have retained the ability to antagonize tetherin. A detailed understanding of $\mathrm{Vpu} /$ tetherin interaction across their transmembrane domains requires further characterization of the involved amino acid residues in the tetherin transmembrane domain and structural determination of the $\mathrm{Vpu} /$ tetherin transmembrane domain complex. It also remains to be determined when and where Vpu initiates interaction with tetherin and how the Vpu/tetherin complex is retained at TGN and early/ recycling endosomes.

SIV Nef SIV strains with the exception of $\operatorname{SIV}_{\mathrm{cpz}}, \mathrm{SIV}_{\mathrm{gor}}$, $\mathrm{SIV}_{\mathrm{gsn}}, \mathrm{SIV}_{\text {mus}}$, and $\mathrm{SIV}_{\text {mon }}$ do not contain a Vpu gene (Gao et al. 1999; Courgnaud et al. 2002, 2003). Vpu of $\mathrm{SIV}_{\mathrm{gsn} / \mathrm{mus} / \mathrm{mon}}$ antagonizes tetherin whereas $\mathrm{SIV}_{\mathrm{cpz} / \mathrm{gor}} \mathrm{Vpu}$ does not overcome tetherin (Sauter et al. 2009). Therefore, SIV should have an alternative strategy to counteract tetherin. Indeed, Nef proteins from SIV strains including $\mathrm{SIV}_{\text {mac }}, \operatorname{SIV}_{\text {smm }}$, SIV $_{\text {blu }}$, and SIV $_{\text {agm }}$ are able to overcome restriction by their respective species tetherin but are unable to overcome human tetherin (Jia et al. 2009; Zhang et al. 2009). Interestingly, Nef proteins from HIV-2 and HIV-1 are also able to counteract Old World monkey tetherins (Rhesus macaque and Sooty mangabey) but are less efficient than SIV Nef (Jia et al. 2009). The sensitivity determinant in simian tetherin to SIV Nef has been mapped to a five-amino-acid region (14-G/DDIWK-18) in the cytoplasmic domain, which is, however, absent in human tetherin (Jia et al. 2009; Zhang et al. 2009). This is in contrast to the role of transmembrane domain of human tetherin in determining its sensitivity to HIV-1 Vpu (Jia et al. 2009; McNatt et al. 2009). Mutating the myristoylation site and cholesterol recognition motif in Nef impairs its tetherin antagonism activity (Jia et al. 2009; Zhang et al. 2009). Additionally, mutations that abrogate CD4 and CD28 downregulation activity of SIVmac239 Nef also diminish the antagonism of Rhesus tetherin, whereas MHCI downregulation is not associated with tetherinantagonizing function (Jia et al. 2009; Zhang et al. 2009). It is believed that SIV Nef protein counteracts simian tetherin by recognizing the "G/DDIWK" motif and downregulating tetherin from the cell surface.

Envelope proteins of HIV-2, SIV, and Ebola virus The envelope glycoproteins (GP) of several viruses have been implicated in tetherin antagonism. A common feature among these tetherin antagonists is that they all seem to target the tetherin ectodomain and cause the relocalization of tetherin from the plasma membrane to the perinuclear region, a strategy that is similar to what $\mathrm{Vpu}$ employs to antagonize human tetherin.

HIV-2 Env Unlike HIV-1, HIV-2 does not encode a vpu gene. Instead, HIV-2 Env fulfills Vpu function to promote virus release (Bour et al. 1996, 2003; Bour and Strebel 1996, 2003). Further studies demonstrate that HIV- $2_{\text {RodA/Rod } 10}$ Env accomplishes this function also by counteracting tetherin (Le Tortorec and Neil 2009; Hauser et al. 2010). Yet this activity of HIV-2 envelope protein seems to be celltype dependent, since the endogenous tetherin in HeLa cells but not the ectopically expressed tetherin in 293Tcells is counteracted by HIV-2 Env. It is noted that HIV-1 Env and SIV $_{\text {mac }}$ Env do not exhibit antitetherin activity (Le Tortorec and Neil 2009). The endocytic motif GYxx $\theta$ within the cytoplasmic tail as well as the extracellular motifs determines the antagonism activity of HIV-2 Env toward human tetherin (Le Tortorec and Neil 2009). In HIV-2 Rod10 Env, a mutation in the membrane-proximal motif Y707 is sufficient to abrogate its antitetherin activity (Hauser et al. 2010). A point mutation A100D in the ectodomain of human tetherin is sufficient to render tetherin resistant to HIV-2 Env; however, both Ebola GP and HIV-1 Vpu were able to antagonize this mutant (Lopez et al. 2010). Tetherin was additionally shown to co-immunoprecipitate with HIV-2 Env likely through interaction between their extracellular domains, and tetherin was consequently downregulated from the cell surface (Le Tortorec and Neil 2009). HIV-2 Env does not cause intracellular depletion of tetherin but does lead to sequestration of tetherin at the TGN (Le Tortorec and Neil 2009). Additionally, Env from another isolate, HIV- $2_{\text {ROD14}}$, is unable to enhance viral release in cells 


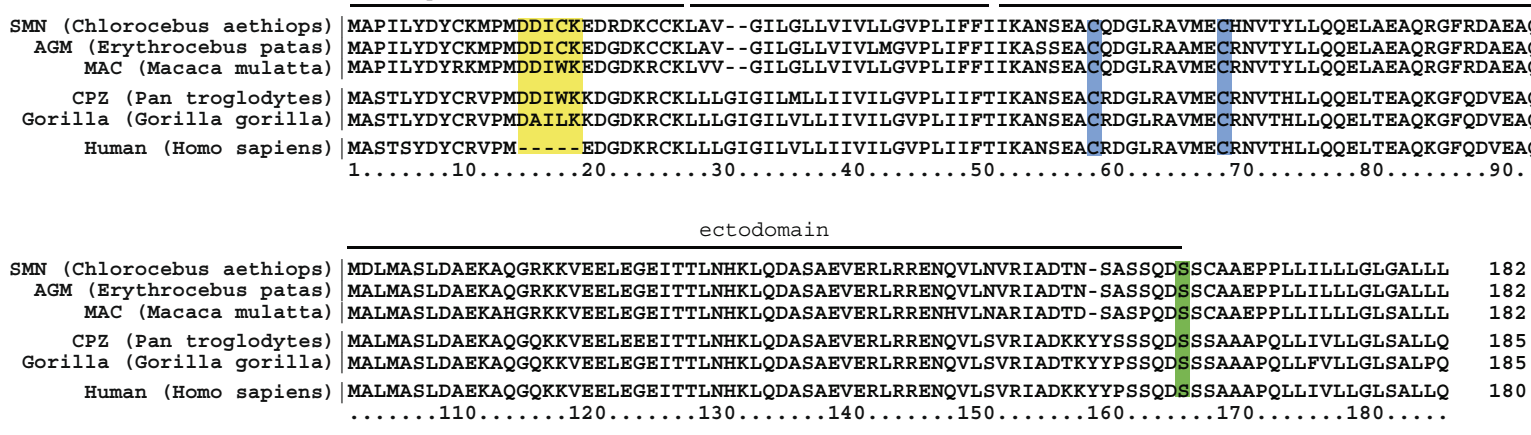

Fig. 5 Sequence alignment of primate tetherins. Aligned are tetherin amino acid sequences of selected simians from the Old World monkey lineage ( $S M N$ Sooty mangabey; $A G M$ African green monkey; $M A C$ Rhesus macaque) and hominid lineage ( $C P Z$ chimpanzee; gorilla; human). Highlighted are the domains that determine sensitivity to Nef (yellow), the cysteines that are involved in tetherin dimerization via disulfide bonds (blue), and the GPI anchor attachment site (green). Alignment was created using ClustalX software; sequence files are derived from GenBank: SMN, ADI58600.1; AGM, ADI58599.1; MAC, ADI58602.1; CPZ, ADI58593.1; Gorilla, ADI58594.1; Human, NP 004326.1

tetherin downregulation (Lopez et al. 2010). Overall, Ebola GP appears to overcome a broad spectrum of tetherin via a mechanism that is distinct from that shared by the Env of HIV-2 and $\mathrm{SIV}_{\tan }$.

KSHV K5 KSHV expresses two RING-CH (MARCH) E3 ubiquitin ligases called K3/MIR1 and K5/MIR2. K5 has long been known to downregulate a number of cell surface proteins including MHC-I (Coscoy and Ganem 2000; Ishido et al. 2000; Stevenson et al. 2000). In a proteomics screening, tetherin was identified as a target of K5 downregulation from the cell surface (Bartee et al. 2006). Subsequent to the discovery of tetherin as a viral restriction factor, K5 was reported to overcome tetherin restriction and restore viral release (Mansouri et al. 2009; Pardieu et al. 2010). K5 exerts its antagonism activity by downregulating tetherin from the cell surface (Mansouri et al. 2009; Pardieu et al. 2010). This activity of $\mathrm{K} 5$ requires its RING domain that mediates the ubiquitination of the lysine residue at position 18 within tetherin cytoplasmic tail (Pardieu et al. 2010). This ubiquitinated tetherin is then sorted into CD63positive late endosomal compartment for degradation (Pardieu et al. 2010). A dominant negative form of VPS4 prevents cell surface depletion of tetherin by K5 (Pardieu et al. 2010). Since this VPS4 mutant disrupts the multivesicular body (MVB) pathway, the results suggest that K5 redirects tetherin from the cell surface through the MVB pathway to late endosomes for degradation.

\section{Tetherin is adapted to viral antagonists and shaped HIV evolution}

Tetherin and other host cell restriction factors have coevolved with viruses. The selective pressure from viruses has lead to species specificity and diversity in restriction 2009; Lopez et al. 2010), nor does it require cell surface 
factors with common ancestors; a sequence alignment of primate tetherins is shown in Fig. 5. Tetherin has also evolved under positive selection by viral antagonists (McNatt et al. 2009; Sauter et al. 2009; Lim et al. 2010). Evolutionary analysis revealed a positive selection at amino acid position 9 in the cytosolic domain of New World monkey tetherin; this was not seen with tetherins of Old World monkeys and hominids (Lim et al. 2010). Instead, tetherin amino acid position 17 has undergone positive selection in Old World monkeys and hominids, in addition to a deletion of five amino acids surrounding this position (14-DDIWK-18) in human tetherin (Lim et al. 2010). It is believed that the positive selection has been driven by an ancient Nef-like antagonist of tetherin (Lim et al. 2010). Interestingly, $\mathrm{Vpu}$ does not seem to have impacted on tetherin evolution in Old World monkeys or hominids, but this might be due to the short period of time since the gainof-function evolution in Vpu (Lim et al. 2010).

HIV-1 was originated by cross-species transmission of SIV from chimpanzees to humans (Gao et al. 1999). $\operatorname{SIV}_{\mathrm{cpz}}$ is considered to have evolved from a recombination of two SIV strains, $\operatorname{SIV}_{\mathrm{gsn}}$ and $\mathrm{SIV}_{\mathrm{rcm}}$. $\mathrm{SIV}_{\mathrm{gsn}}$ but not $\mathrm{SIV}_{\mathrm{rcm}}$ encodes Vpu (Courgnaud et al. 2002, 2003; Dazza et al. 2005). $\operatorname{SIV}_{\mathrm{cpz}}$ obtained Vpu from $\operatorname{SIV}_{\mathrm{gsn}}$ and Nef from $\mathrm{SIV}_{\mathrm{rcm}}$. It is speculated that, in the original $\mathrm{SIV}_{\mathrm{cpz}}, \mathrm{Vpu}$ and Nef proteins had only little antitetherin capacity (Sauter et al. 2009; Yang et al. 2010b). Over time, SIV $_{\mathrm{cpz}}$ Nef evolved to become the primary tetherin antagonist, while $\mathrm{Vpu}$ maintained the capacity to downmodulate CD4 from the cell surface (Sauter et al. 2009; Yang et al. 2010b). When $\mathrm{SIV}_{\mathrm{cpz}}$ crossed the species barrier to infect humans, Nef was unable to antagonize human tetherin due to the lack of the Nef-sensitive ${ }_{14}$ DDIWK $_{18}$ site. Vpu subsequently (re) gained its tetherin-antagonizing function (Sauter et al. 2009; Zhang et al. 2009; Lim et al. 2010). However, only the Vpu of pandemic HIV-1 group $\mathrm{M}$ efficiently antagonizes human tetherin whereas $\mathrm{Vpu}$ of group $\mathrm{N}$ and $\mathrm{O}$ is a poor tetherin antagonist (Sauter et al. 2009). This suggests that the extent of $\mathrm{Vpu}$ adaptation to antagonize human tetherin influences the pathogenicity of HIV-1.

In contrast to $\operatorname{SIV}_{\mathrm{cpz}}$, the $\mathrm{SIV}_{\mathrm{smm}}$ strain that gave rise to HIV-2 lacks vpu (Gao et al. 1992; Chen et al. 1997). HIV-2 overcame human tetherin restriction through evolving its envelope protein into a tetherin antagonist (Le Tortorec and Neil 2009). This adaptation, however, might involve a fitness cost, which is reflected by the low infectivity of HIV-2 when compared to HIV-1. Nonetheless, this flexibility in viral strategy to antagonize tetherin reflects the intense selective pressure exerted by tetherin during the adaptation of the HIV-1 and HIV-2 precursor viruses to the new host. Considering the high conservation of tetherin in various species and its restrictive nature over a wide range of enveloped viruses, the example of shaping HIV-1 and
HIV-2 evolution suggests a highly active role of tetherinmediated innate immunity against cross-species transmission of viruses beyond HIV (McNatt et al. 2009; Sauter et al. 2009; Lim et al. 2010; Yang et al. 2010b).

\section{Concluding remarks}

Since the discovery of the potent antiviral activity of tetherin, efforts are already underway to exploit Vpu/tetherin interaction as a new target to develop novel anti-HIV-1 therapeutics such as small compounds that block Vpu function and thus expose HIV-1 to tetherin restriction. Similar approaches have been proven successful in the development of small molecule inhibitors of Vif/APOBEC3G interaction (Nathans et al. 2008; Cen et al. 2010). It has already been reported that a Vpu-targeting drug BIT225 inhibited the release of HIV-1 from macrophages (Khoury et al. 2010). However, protease inhibitors, which modulate transmembrane transporters and thereby might also interfere with $\mathrm{Vpu}$, proved not to impact on tetherin cell surface expression or Vpu-mediated downmodulation of tetherin (Kuhl et al. 2010a).

It is noted that the anti-HIV-1 activity of tetherin and the $\mathrm{Vpu}$ antagonism have been investigated thus far mainly in cultured cell lines including HeLa, 293T, COS-7, HT1080, CEMx174, H9, and SupT1 cells. The results thus obtained need to be verified by experiments in primary human immune cells such as CD4 $+\mathrm{T}$ cells and macrophages partially because some observations have been cell-typespecific. For instance, although HIV-1 replication is Vpuresponsive in CEMx174 and $\mathrm{H} 9$ cells, the cell surface level of tetherin is not affected by Vpu expression as opposed to the downregulation of cell surface tetherin by $\mathrm{Vpu}$ in HeLa and 293T cells (Miyagi et al. 2009; Van Damme et al. 2008). The physiological role of tetherin in the pathogenesis of HIV-1 and other enveloped viruses also remains to be determined in animal models. Although tetherin potently blocks the release of virus particles from cell surface and thus restricts dissemination of free virus particles, further investigation is needed to clarify its role in cell-to-cell virus transmission that is much more efficient than transmission by free virus particles.

Acknowledgements This work was supported by funds of Canadian Institutes of Health Research to C.L.

Conflict of Interest There is no conflict of interest for any of the authors.

Open Access This article is distributed under the terms of the Creative Commons Attribution Noncommercial License which permits any noncommercial use, distribution, and reproduction in any medium, provided the original author(s) and source are credited. 


\section{References}

Abada P, Noble B, Cannon PM (2005) Functional domains within the human immunodeficiency virus type 2 envelope protein required to enhance virus production. J Virol 79:3627-3638

Anderson JL, Hope TJ (2004) HIV accessory proteins and surviving the host cell. Curr HIV/AIDS Rep 1:47-53

Andrew AJ, Miyagi E, Kao S, Strebel K (2009) The formation of cysteine-linked dimers of BST-2/tetherin is important for inhibition of HIV-1 virus release but not for sensitivity to Vpu. Retrovirology 6:80

Bartee E, McCormack A, Früh K (2006) Quantitative membrane proteomics reveals new cellular targets of viral immune modulators. PLoS Pathog 2:e107

Blasius AL, Giurisato E, Cella M, Schreiber RD, Shaw AS, Colonna M (2006) Bone marrow stromal cell antigen 2 is a specific marker of type I IFN-producing cells in the naive mouse, but a promiscuous cell surface antigen following IFN stimulation. J Immunol 177:3260-3265

Bour S, Strebel K (1996) The human immunodeficiency virus (HIV) type 2 envelope protein is a functional complement to HIV type 1 $\mathrm{Vpu}$ that enhances particle release of heterologous retroviruses. J Virol 70:8285-8300

Bour S, Strebel K (2003) The HIV-1 Vpu protein: a multifunctional enhancer of viral particle release. Microbes Infect 5:1029-1039

Bour S, Schubert U, Peden K, Strebel K (1996) The envelope glycoprotein of human immunodeficiency virus type 2 enhances viral particle release: a Vpu-like factor? J Virol 70:820-829

Bour S, Akari H, Miyagi E, Strebel K (2003) Naturally occurring amino acid substitutions in the HIV-2 ROD envelope glycoprotein regulate its ability to augment viral particle release. Virology 309:85-98

Brown D, Trowsdale J, Allen R (2004) The LILR family: modulators of innate and adaptive immune pathways in health and disease. Tissue Antigens 64:215-225

Cao W, Rosen DB, Ito T, Bover L, Bao M, Watanabe G, Yao Z, Zhang L, Lanier LL, Liu Y-J (2006) Plasmacytoid dendritic cell-specific receptor ILT7-Fc epsilon RI gamma inhibits Toll-like receptorinduced interferon production. J Exp Med 203:1399-1405

Cao W, Bover L, Cho M, Wen X, Hanabuchi S, Bao M, Rosen DB, Wang Y-H, Shaw JL, Du Q, Li C, Arai N, Yao Z, Lanier LL, Liu Y-J (2009) Regulation of TLR7/9 responses in plasmacytoid dendritic cells by BST2 and ILT7 receptor interaction. J Exp Med 206:1603-1614

Casartelli N, Sourisseau M, Feldmann J, Guivel-Benhassine F, Mallet A, Marcelin A-G, Guatelli J, Schwartz O (2010) Tetherin restricts productive HIV-1 cell-to-cell transmission. PLoS Pathog 6: e1000955

Cen S, Peng Z-G, Li X-Y, Li Z-R, Ma J, Wang Y-M, Fan B, You X-F, Wang Y-P, Liu F, Shao R-G, Zhao L-X, Yu L, Jiang J-D (2010) Small molecular compounds inhibit HIV-1 replication through specifically stabilizing APOBEC3G. J Biol Chem 285:1654616552

Chen Z, Luckay A, Sodora DL, Telfer P, Reed P, Gettie A, Kanu JM, Sadek RF, Yee J, Ho DD, Zhang L, Marx PA (1997) Human immunodeficiency virus type 2 (HIV-2) seroprevalence and characterization of a distinct HIV-2 genetic subtype from the natural range of simian immunodeficiency virus-infected sooty mangabeys. J Virol 71:3953-3960

Coscoy L, Ganem D (2000) Kaposi's sarcoma-associated herpesvirus encodes two proteins that block cell surface display of MHC class I chains by enhancing their endocytosis. Proc Natl Acad Sci USA 97:8051-8056

Courgnaud V, Abela B, Pourrut X, Mpoudi-Ngole E, Loul S, Delaporte E, Peeters M (2003) Identification of a new simian immunodeficiency virus lineage with a vpu gene present among different Cercopithecus monkeys (C. mona, C. cephus, and C. nictitans) from Cameroon. J Virol 77:12523-12534

Courgnaud V, Salemi M, Pourrut X, Mpoudi-Ngole E, Abela B, Auzel P, Bibollet-Ruche F, Hahn B, Vandamme A-M, Delaporte E, Peeters M (2002) Characterization of a novel simian immunodeficiency virus with a vpu gene from greater spot-nosed monkeys (Cercopithecus nictitans) provides new insights into simian/ human immunodeficiency virus phylogeny. J Virol 76:82988309

Dazza M-C, Ekwalanga M, Nende M, Shamamba KB, Bitshi P, Paraskevis D, Saragosti S (2005) Characterization of a novel vpu-harboring simian immunodeficiency virus from a Dent's Mona monkey (Cercopithecus mona denti). J Virol 79:8560 8571

Douglas JL, Viswanathan K, McCarroll MN, Gustin JK, Früh K, Moses AV (2009) Vpu directs the degradation of the human immunodeficiency virus restriction factor BST-2/tetherin via a \{beta\} TrCP-dependent mechanism. J Virol 83:7931-7947

Douville RN, Hiscott J (2010) The interface between the innate interferon response and expression of host retroviral restriction factors. Cytokine 52:108-115

Dubé M, Roy BB, Guiot-Guillain P, Binette J, Mercier J, Chiasson A, Cohen EA (2010) Antagonism of tetherin restriction of HIV-1 release by $\mathrm{Vpu}$ involves binding and sequestration of the restriction factor in a perinuclear compartment. PLoS Pathog 6: e1000856

Fitzpatrick K, Skasko M, Deerinck TJ, Crum J, Ellisman MH, Guatelli J (2010) Direct restriction of virus release and incorporation of the interferon-induced protein BST-2 into HIV-1 particles. PLoS Pathog 6:e1000701

Gao F, Yue L, White AT, Pappas PG, Barchue J, Hanson AP, Greene BM, Sharp PM, Shaw GM, Hahn BH (1992) Human infection by genetically diverse SIVSM-related HIV-2 in West Africa. Nature 358:495-499

Gao F, Bailes E, Robertson DL, Chen Y, Rodenburg CM, Michael SF, Cummins LB, Arthur LO, Peeters M, Shaw GM, Sharp PM, Hahn BH (1999) Origin of HIV-1 in the chimpanzee Pan troglodytes troglodytes. Nature 397:436-441

Goffinet C, Allespach I, Homann S, Tervo H-M, Habermann A, Rupp D, Oberbremer L, Kern C, Tibroni N, Welsch S, Krijnse-Locker J, Banting G, Krausslich H-G, Fackler OT, Keppler OT (2009) HIV-1 antagonism of CD317 is species specific and involves Vpu-mediated proteasomal degradation of the restriction factor. Cell Host Microbe 5:285-297

Goto T, Kennel SJ, Abe M, Takishita M, Kosaka M, Solomon A, Saito S (1994) A novel membrane antigen selectively expressed on terminally differentiated human B cells. Blood 84:1922-1930

Göttlinger HG, Dorfman T, Cohen EA, Haseltine WA (1993) Vpu protein of human immunodeficiency virus type 1 enhances the release of capsids produced by gag gene constructs of widely divergent retroviruses. Proc Natl Acad Sci USA 90:7381-7385

Gupta RK, Hué S, Schaller T, Verschoor E, Pillay D, Towers GJ, Hope TJ (2009a) Mutation of a single residue renders human tetherin resistant to HIV-1 Vpu-mediated depletion. PLoS Pathog 5: e1000443

Gupta RK, Mlcochova P, Pelchen-Matthews A, Petit SJ, Mattiuzzo G, Pillay D, Takeuchi Y, Marsh M, Towers GJ (2009b) Simian immunodeficiency virus envelope glycoprotein counteracts tetherin/BST-2/CD317 by intracellular sequestration. Proc Natl Acad Sci USA 106:20889-20894

Habermann A, Krijnse Locker J, Oberwinkler H, Eckhardt M, Homann S, Andrew A, Strebel K, Krausslich H-G (2010) CD317/tetherin is enriched in the HIV-1 envelope and downregulated from the plasma membrane upon virus infection. J Virol 84:4646-4658 
Hauser H, Lopez LA, Yang SJ, Oldenburg JE, Exline CM, Guatelli JC, Cannon PM (2010) HIV-1 Vpu and HIV-2 Env counteract BST-2/tetherin by sequestration in a perinuclear compartment. Retrovirology 7:51

Hegde RS, Mastrianni JA, Scott MR, DeFea KA, Tremblay P, Torchia M, DeArmond SJ, Prusiner SB, Lingappa VR (1998) A transmembrane form of the prion protein in neurodegenerative disease. Science 279:827-834

Henriet S, Mercenne G, Bernacchi S, Paillart J-C, Marquet R (2009) Tumultuous relationship between the human immunodeficiency virus type 1 viral infectivity factor (Vif) and the human APOBEC-3G and APOBEC-3F restriction factors. Microbiol Mol Biol Rev 73:211-232

Hinz A, Miguet N, Natrajan G, Usami Y, Yamanaka H, Renesto P, Hartlieb B, Mccarthy AA, Simorre J-P, Göttlinger H, Weissenhorn W (2010) Structural basis of HIV-1 tethering to membranes by the BST-2/tetherin ectodomain. Cell Host Microbe 7:314-323

Ishido S, Wang C, Lee BS, Cohen GB, Jung JU (2000) Downregulation of major histocompatibility complex class I molecules by Kaposi's sarcoma-associated herpesvirus $\mathrm{K} 3$ and $\mathrm{K} 5$ proteins. J Virol 74:5300-5309

Ishikawa J, Kaisho T, Tomizawa H, Lee BO, Kobune Y, Inazawa J, Oritani K, Itoh M, Ochi T, Ishihara K (1995) Molecular cloning and chromosomal mapping of a bone marrow stromal cell surface gene, BST2, that may be involved in pre-B-cell growth. Genomics 26:527-534

Iwabu Y, Fujita H, Kinomoto M, Kaneko K, Ishizaka Y, Tanaka Y, Sata T, Tokunaga K (2009) HIV-1 accessory protein Vpu internalizes cell-surface BST-2/tetherin through transmembrane interactions leading to lysosomes. J Biol Chem 284:35060 35072

Jia B, Serra-Moreno R, Neidermyer W, Rahmberg A, Mackey J, Fofana IB, Johnson WE, Westmoreland S, Evans DT, Emerman M (2009) Species-specific activity of SIV Nef and HIV-1 Vpu in overcoming restriction by tetherin/BST2. PLoS Pathog 5: e1000429

Jolly C, Sattentau QJ (2005) Human immunodeficiency virus type 1 virological synapse formation in $\mathrm{T}$ cells requires lipid raft integrity. J Virol 79:12088-12094

Jolly C, Booth NJ, Neil SJD (2010) Cell-cell spread of human immunodeficiency virus type-1 overcomes tetherin/BST-2 mediated restriction in T cells. J Virol 84:12185-12199

Jouvenet N, Neil SJD, Zhadina M, Zang T, Kratovac Z, Lee Y, Mcnatt M, Hatziioannou T, Bieniasz PD (2009) Broad-spectrum inhibition of retroviral and filoviral particle release by tetherin. J Virol 83:1837-1844

Kaletsky RL, Francica JR, Agrawal-Gamse C, Bates P (2009) Tetherin-mediated restriction of filovirus budding is antagonized by the Ebola glycoprotein. Proc Natl Acad Sci USA 106:28862891

Kawai T, Akira S (2006) Innate immune recognition of viral infection. Nat Immunol 7:131-137

Khoury G, Ewart G, Luscombe C, Miller M, Wilkinson J (2010) Antiviral efficacy of the novel compound BIT225 against HIV-1 release from human macrophages. Antimicrob Agents Chemother 54:835-845

Kuhl BD, Sloan RD, Donahue DA, Bar-Magen T, Liang C, Wainberg MA (2010a) Neither ritonavir nor darunavir affect cell surface expression of tetherin or Vpu-mediated tetherin downmodulation. AIDS 24:1970-1972

Kuhl BD, Sloan, Richard D, Donahue Daniel A, Bar-Magen, Tamara, Linag, Chen, Wainberg, Mark A (2010b) Tetherin restricts direct cellcell viral transfer and transmission of HIV-1. Retrovirology 7:115

Kupzig S, Korolchuk V, Rollason R, Sugden A, Wilde A, Banting G (2003) Bst-2/HM1.24 is a raft-associated apical membrane protein with an unusual topology. Traffic 4:694-709
Le Tortorec A, Neil SJD (2009) Antagonism to and intracellular sequestration of human tetherin by the human immunodeficiency virus type 2 envelope glycoprotein. J Virol 83:11966-11978

Lim ES, Malik HS, Emerman M (2010) Ancient adaptive evolution of tetherin shaped the functions of $\mathrm{Vpu}$ and Nef in human immunodeficiency virus and primate lentiviruses. J Virol 84:7124-7134

Lopez LA, Yang SJ, Hauser H, Exline CM, Haworth KG, Oldenburg J, Cannon PM (2010) Ebola virus glycoprotein counteracts BST$2 /$ tetherin restriction in a sequence-independent manner that does not require tetherin surface removal. J Virol 84:7243-7255

Malim MH, Emerman M (2008) HIV-1 accessory proteins-ensuring viral survival in a hostile environment. Cell Host Microbe 3:388398

Mangeat B, Gers-Huber G, Lehmann M, Zufferey M, Luban J, Piguet V (2009) HIV-1 Vpu neutralizes the antiviral factor tetherin/BST2 by binding it and directing its beta-TrCP2-dependent degradation. PLoS Pathog 5:e1000574

Mansouri M, Viswanathan K, Douglas JL, Hines J, Gustin J, Moses AV, Früh K (2009) Molecular mechanism of BST2/tetherin downregulation by K5/MIR2 of Kaposi's sarcoma-associated herpesvirus. J Virol 83:9672-9681

Martin N, Sattentau Q (2009) Cell-to-cell HIV-1 spread and its implications for immune evasion. Curr Opin HIV AIDS 4:143149

Masuyama N, Kuronita T, Tanaka R, Muto T, Hirota Y, Takigawa A, Fujita H, Aso Y, Amano J, Tanaka Y (2009) HM1.24 is internalized from lipid rafts by clathrin-mediated endocytosis through interaction with adaptin. J Biol Chem 284:15927-15941

McNatt MW, Zang T, Hatziioannou T, Bartlett M, Fofana IB, Johnson WE, Neil SJD, Bieniasz PD, Hope TJ (2009) Species-specific activity of HIV-1 Vpu and positive selection of tetherin transmembrane domain variants. PLoS Pathog 5:e1000300

Mitchell RS, Katsura C, Skasko MA, Fitzpatrick K, Lau D, Ruiz A, Stephens EB, Margottin-Goguet F, Benarous R, Guatelli JC (2009) Vpu antagonizes BST-2-mediated restriction of HIV-1 release via beta-TrCP and endo-lysosomal trafficking. PLoS Pathog 5:e1000450

Miyagi E, Andrew AJ, Kao S, Strebel K (2009) Vpu enhances HIV-1 virus release in the absence of Bst-2 cell surface downmodulation and intracellular depletion. Proc Natl Acad Sci USA 106:2868-2873

Miyakawa K, Ryo A, Murakami T, Ohba K, Yamaoka S, Fukuda M, Guatelli J, Yamamoto N (2009) BCA2/Rabring7 promotes tetherin-dependent HIV-1 restriction. PLoS Pathog 5:e1000700

Nathans R, Cao H, Sharova N, Ali A, Sharkey M, Stranska R, Stevenson M, Rana TM (2008) Small-molecule inhibition of HIV-1 Vif. Nat Biotechnol 26:1187-1192

Neil SJD, Zang T, Bieniasz PD (2008) Tetherin inhibits retrovirus release and is antagonized by HIV-1 Vpu. Nature 451:425430

Neil SJD, Sandrin V, Sundquist WI, Bieniasz PD (2007) An interferon-alpha-induced tethering mechanism inhibits HIV-1 and Ebola virus particle release but is counteracted by the HIV$1 \mathrm{Vpu}$ protein. Cell Host Microbe 2:193-203

Niewiadomska AM, Yu X-F (2009) Host restriction of HIV-1 by APOBEC3 and viral evasion through Vif. Curr Top Microbiol Immunol 339:1-25

Ohtomo T, Sugamata Y, Ozaki Y, Ono K, Yoshimura Y, Kawai S, Koishihara Y, Ozaki S, Kosaka M, Hirano T, Tsuchiya M (1999) Molecular cloning and characterization of a surface antigen preferentially overexpressed on multiple myeloma cells. Biochem Biophys Res Commun 258:583-591

Ono K, Ohtomo T, Yoshida K, Yoshimura Y, Kawai S, Koishihara Y, Ozaki S, Kosaka M, Tsuchiya M (1999) The humanized antiHM1.24 antibody effectively kills multiple myeloma cells by 
human effector cell-mediated cytotoxicity. Mol Immunol 36:387395

Ozaki S, Kosaka M, Wakatsuki S, Abe M, Koishihara Y, Matsumoto T (1997) Immunotherapy of multiple myeloma with a monoclonal antibody directed against a plasma cell-specific antigen, HM1.24. Blood 90:3179-3186

Pais-Correia A-M, Sachse M, Guadagnini S, Robbiati V, Lasserre R, Gessain A, Gout O, Alcover A, Thoulouze M-I (2009) Biofilmlike extracellular viral assemblies mediate HTLV-1 cell-to-cell transmission at virological synapses. Nat Med 16:83-89

Pardieu C, Vigan R, Wilson SJ, Calvi A, Zang T, Bieniasz P, Kellam P, Towers GJ, Neil SJD (2010) The RING-CH ligase K5 antagonizes restriction of KSHV and HIV-1 particle release by mediating ubiquitin-dependent endosomal degradation of tetherin. PLoS Pathog 6:e1000843

Park SH, Mrse AA, Nevzorov AA, Mesleh MF, Oblatt-Montal M, Montal M, Opella SJ (2003) Three-dimensional structure of the channel-forming trans-membrane domain of virus protein " $u$ " (Vpu) from HIV-1. J Mol Biol 333:409-424

Perez-Caballero D, Zang T, Ebrahimi A, Mcnatt MW, Gregory DA, Johnson MC, Bieniasz PD (2009) Tetherin inhibits HIV-1 release by directly tethering virions to cells. Cell 139:499-511

Piguet V, Sattentau Q (2004) Dangerous liaisons at the virological synapse. J Clin Invest 114:605-610

Popik W, Alce TM (2004) CD4 receptor localized to non-raft membrane microdomains supports HIV-1 entry. Identification of a novel raft localization marker in CD4. J Biol Chem 279:704712

Popik W, Alce TM, Au W-C (2002) Human immunodeficiency virus type 1 uses lipid raft-colocalized CD4 and chemokine receptors for productive entry into CD4(+) T cells. J Virol 76:4709-4722

Rollason R, Korolchuk V, Hamilton C, Schu P, Banting G (2007) Clathrin-mediated endocytosis of a lipid-raft-associated protein is mediated through a dual tyrosine motif. J Cell Sci 120:3850-3858

Rollason R, Korolchuk V, Hamilton C, Jepson M, Banting G (2009) A CD317/tetherin-RICH2 complex plays a critical role in the organization of the subapical actin cytoskeleton in polarized epithelial cells. J Cell Biol 184:721-736

Rong L, Zhang J, Lu J, Pan Q, Lorgeoux R-P, Aloysius C, Guo F, Liu S-L, Wainberg MA, Liang C (2009) The transmembrane domain of BST-2 determines its sensitivity to down-modulation by human immunodeficiency virus type 1 Vpu. J Virol 83:75367546

Sadler AJ, Williams BRG (2008) Interferon-inducible antiviral effectors. Nat Rev Immunol 8:559-568

Sakuma T, Noda T, Urata S, Kawaoka Y, Yasuda J (2009) Inhibition of Lassa and Marburg virus production by tetherin. J Virol 83:2382-2385

Sato K, Yamamoto SP, Misawa N, Yoshida T, Miyazawa T, Koyanagi Y (2009) Comparative study on the effect of human BST-2/ tetherin on HIV-1 release in cells of various species. Retrovirology 6:53

Sattentau Q (2008) Avoiding the void: cell-to-cell spread of human viruses. Nat Rev Microbiol 6:815-826
Sauter D et al (2009) Tetherin-driven adaptation of Vpu and Nef function and the evolution of pandemic and nonpandemic HIV-1 strains. Cell Host Microbe 6:409-421

Schubert HL, Zhai Q, Sandrin V, Eckert DM, Garcia-Maya M, Saul L, Sundquist WI, Steiner RA, Hill CP (2010) Structural and functional studies on the extracellular domain of BST2/tetherin in reduced and oxidized conformations. Proc Natl Acad Sci USA 107:17951-17956

Stevenson PG, Efstathiou S, Doherty PC, Lehner PJ (2000) Inhibition of MHC class I-restricted antigen presentation by gamma 2herpesviruses. Proc Natl Acad Sci USA 97:8455-8460

Stewart RS, Drisaldi B, Harris DA (2001) A transmembrane form of the prion protein contains an uncleaved signal peptide and is retained in the endoplasmic reticulum. Mol Biol Cell 12:881-889

Strebel K, Klimkait T, Maldarelli F, Martin MA (1989) Molecular and biochemical analyses of human immunodeficiency virus type 1 vpu protein. J Virol 63:3784-3791

Tokarev AA, Munguia J, Guatelli JC (2010) Serine-threonine ubiquitination mediates downregulation of BST-2/tetherin and relief of restricted virion release by HIV-1 Vpu. J Virol 85:51-63

Van Damme N, Goff D, Katsura C, Jorgenson RL, Mitchell R, Johnson MC, Stephens EB, Guatelli J (2008) The interferoninduced protein BST-2 restricts HIV-1 release and is downregulated from the cell surface by the viral Vpu protein. Cell Host Microbe 3:245-252

Varthakavi V, Smith RM, Bour SP, Strebel K, Spearman P (2003) Viral protein $U$ counteracts a human host cell restriction that inhibits HIV-1 particle production. Proc Natl Acad Sci USA 100:15154-15159

Versteeg GA, García-Sastre A (2010) Viral tricks to grid-lock the type I interferon system. Curr Opin Microbiol 13:508-516

Vidal-Laliena M, Romero X, March S, Requena V, Petriz J, Engel P (2005) Characterization of antibodies submitted to the B cell section of the 8th Human Leukocyte Differentiation Antigens Workshop by flow cytometry and immunohistochemistry. Cell Immunol 236:6-16

Vigan R, Neil SJD (2010) Determinants of tetherin antagonism in the transmembrane domain of the human immunodeficiency virus type-1 (HIV-1) Vpu protein. J Virol 84:12958-12970

Waheed AA, Freed EO (2009) Lipids and membrane microdomains in HIV-1 replication. Virus Res 143:162-176

Yang H, Wang J, Jia X, Mcnatt MW, Zang T, Pan B, Meng W, Wang H-W, Bieniasz PD, Xiong Y (2010a) Structural insight into the mechanisms of enveloped virus tethering by tetherin. Proc Natl Acad Sci USA 107:18428-18432

Yang SJ, Lopez LA, Hauser H, Exline CM, Haworth KG, Cannon PM (2010b) Anti-tetherin activities in Vpu-expressing primate lentiviruses. Retrovirology 7:13

Zhang F, Wilson SJ, Landford WC, Virgen B, Gregory D, Johnson MC, Munch J, Kirchhoff F, Bieniasz PD, Hatziioannou T (2009) Nef proteins from simian immunodeficiency viruses are tetherin antagonists. Cell Host Microbe 6:54-67

Zhang J, Liang C (2010) BST-2 diminishes HIV-1 infectivity. J Virol $84: 12336-12343$ 